\title{
Design of Information Integration Architecture using SOA
}

\author{
C.Punitha Devi, \\ Department of Computer \\ Science and Engineering, \\ Pondicherry University, \\ Puducherry, India
}

\author{
V. Prasanna Venkatesan \\ Department of Banking \\ Technology, \\ Pondicherry University, \\ Puducherry, India
}

\author{
S.Diwahar, \\ Department of Computer \\ Science and Engineering, \\ Pondicherry University, \\ Puducherry, India
}

\author{
G.Shanmugasundaram \\ Department of Information Technology, \\ Sri Manakula Vinayagar Engineering College, \\ Puducherry, India
}

\begin{abstract}
Information integration challenges observed from the literature review indicates that it needs to be addressed at the architectural level rather at the techniques or approach. The most significant challenge considered thereafter is to deliver a better technical methodology to facilitate an open architecture with standard interfaces to aid in information integration, interoperability and retrieval. One way to unlock the information from its containers by separating the implementation and interface is achieved through SOA. This work aims to design architecture for information integration using SOA to obtain integrated information as a service. The layers and components strive to provide all aspects of information integration in order to give complete picture. An explanation pertaining to the layers are based on roles and responsibilities and the components are based on a template. Discussion part exhibits the proposed architecture's qualities on comparing with models specified in the related work.
\end{abstract}

\section{Keywords}

Information Integration, SOA, Architecture, Service Oriented Integration

\section{INTRODUCTION}

Talking about Data issues, thought was only in terms of volume, but the rate at which it has grown has lead to be thought of as complexity issue. Information sources ranges from social media to mobile phones, RFID tags, smart devices and the web. In this environment business and technical users need access to data and derive insights to sustain competitive edge. Access to the information which lies in different forms and geographically distributed becomes a common problem where the complexity of retrieving the information is hidden from the user by providing a single view of the sources. Information and their request also appear to be in different forms. Processing the request to correctly interpret the information needed, integration of information sources to provide a single view and availing the information with respect to the request has been recognized as a business requirement. Various Integration strategies are already adopted and analyzed but one recent strategy that has taken predominance is Service Oriented Architecture (SOA).
SOA is an architectural style that supports service orientation. Services are the primary software constituents of the architecture where it corresponds to basic functionalities of the architecture. SOA success and adoption is primarily due to its suitability for integration. Integration has taken a new shape with the evolution of SOA as it supports flexibility, scalability and reusability, which have become significant factors of integration software. The service principles also help in achieving it. This paper highlights a model for information integration with SOA. The organization of this paper is as follows. Next section specifies the related work on information integration models, then the proposed model, discussion and conclusion.

\section{RELATED WORK}

Information integration is the most important concept that has been ruling business and technology in the recent time. Various solutions have been provided for information integration. For a long period it was addressed as Enterprise Information Integration [1][2]. Its scope was very limited as the solutions had been biased in providing integration either application specific [3][4] or at the data integration level [5][6][7] or considering semantics, ontology [8][9][10][11] or addressing specific information types or sources [12][13] and Industry oriented model [23][24]. This is the outline of the details specified under this section. Information integration solutions exist in many forms such as approaches, frameworks, architectures, and models. The related work considers all these representations to be in general as models and analyzes for their limitations.

\subsection{Information Sources Specific}

The existing models are based on certain requirements. They are designed to solve the requirements. One of the major such requirement is handling web sources. Certain models are built considering the access to information to web sources as their primary requirement and designed the model appropriately [12][13]. The methodology and technology adopted however differs. [12] has used machine learning and agents whereas the other [13] has semantics as its base. One model [14] has considered adding geospatial and temporal data as unique feature of its proposal. This model has concentrated majorly on the Query processing for addressing heterogeneity in various domains. [15][16] describes a model that considers 
integration of legacy system information as its main requirement and provides a model with wrappers and adapters in a web service based environment.

\subsection{Automation (semantic and ontology based)}

Information integration was realized as one step above data integration. Data integration was understood to be the underlying aspect of information integration with the realization of semantics and ontology. Data integration along with usage of semantics and ontology for information extraction and contextual binding were termed as information integration. Models of such systems were completely biased in solving issues related to semantics and ontologies. [8] The architecture considers the data to be made available as service, the retrieved data service is passed on to the semantics and ontology through an integration engine which proceeds with the help of logic rules. [9] This provides ontology based information where a layered architecture is adopted for information integration. The layers specify the core functionalities communication, core, and data. Agents are used to bring out the needed functionalities. Information is the main objective rather than the process and is based on a semantic model for information sharing. Web sources are the mentioned type of sources but its design is suitable to add any sources with implementation types specified. [10] Gives a model for enterprise information integration in a web service environment, where semantics at the data level and service level are stated for solving gaps of distributed computing. The primary concern of this model is to reduce human intervention and automate the integration process through semantic web services. [11] Explains a model which consists of Data resource layer, Service implementation layer, Application system layer and User interface layer. Again it has also taken semantics at the service level as the significant challenge and proposed architecture for it. The domain ontology, its representation semantic tools, and etc., are the main features used to solve service implementation followed by service composition and decomposition giving service oriented integration, the principle behind this architecture.

\subsection{Global view models}

Some models have attempted to give a global view of the information integration process. One such is brought out in [17], where a workflow is stated with stages as Input, Planning, Execution and Output. A workflow management system, which gives the automated generation of workflow in Planning stage, data flow, control flow and exception flow of information integration in execution stage in a web services environment is clearly explained with a hospital case study. The conceptual model and the corresponding implementation model has also been specified. Loglo [18] is an architecture that integrates distributed heterogeneous information. This model specifies itself as SOA based integration and model is also of request response principle through messages.

\subsection{Application specific models}

Information integration lies across all domains and in every business. Integrated information cuts across all departments of a business or domain from production to finance, human resource, stock maintenance, etc. For providing solutions to such specific issues certain models exist. Examples of such models are specified in [3][4]. These models are focused in framing the applications need like information integration for inline accommodation, which strive to solve issues such as handling information across the internet and dynamic inclusion of information sources with respect to accommodation. Publish-combine-use process is adopted for a semantic, ontology based model. Likewise an information integration framework is proposed for personalized information in higher education. A layered approach is followed with communication, web services and semantic layers along with the information sources. The concentration is greater in the web service layer and in handling semantics. Another domain where information integration has been a principle need is health and financial domain. Several models for different applications of these domains for information integration has been researched [19][20][21].

\subsection{Industry specific models}

Software industry has its own growth in research towards information integration. As said it was widely called as EII. Industry and academic had a common view on definition of EII. This thought was taken over by virtualization. Even today data virtualization is the core in case of many data solution providing enterprises like

Informatica, Composite, Oracle, Microsoft, etc [22]. Normally some topics would have a wide reach in case of industry but not academic. One such research area is service oriented architecture. These enterprises along with others such as IBM, redhat, Accenture all have adopted SOA as the design style for framing the solutions. They provide Data services which they claim it to be information as service [22]. Hence the integration is achieved through Data virtualization with SOA. Forrester has coined a new term for this "Information fabric" which gives all these functionalities with the prime objective as data virtualization [23]. A reference architecture is specified which gives a complete picture for data virtualization, where information as a service is a partial outcome of the model.

\subsection{Limitations}

The different models are grouped under their common features and explained. It could be recognized that information integration has achieved new heights in approaches, or models or architectures or frameworks in the recent past due to increase in different types of information sources and volume of information. However still there has been discussions as to whether the existing research has accomplished all of its features in complete [24][25][26]. The limitations that are evident from the existing research are

- Model confines to specific requirements

- No proper definition or standard or methodology to formulate the information request and result after integration

- Integration is considered as to provide a single view of the information sources only i.e., Data modeling layer only.

- Even though the models are generically specified but concentration lies to any one layer of the design

- Pertain to issues of specific information sources, and does not cover all information sources.

- Top level view of the complete process is not been addressed

- Different tools are present for each unit of integration, no tool for the complete integration

- No proper chaining of these tools to assist in appropriate usage

- Recent solutions have started to grow domain and application intended. 


\section{THE PROPOSED ARCHITECTURE}

In [27] has shown the different definitions of information integration and has proposed a new characterization of information integration addressing input, process and output aspects of it. As per this proposed characterization information integration does not stop in providing a single view of the information sources but consists of a series of sub process, and a listing of these sub process for their dependency.

Input: The request, request handling, interpretation of request with respect to domain ontology, to analyze the request for the need of different information.

Process: Retrieval of the all associated data for it be modeled into information with semantics, ontology, and data sources rules, integration of the various information

Output: Analysis of the requestor for accessibility rights, security aspects, structuring of the integrated result with respect to the requestor.

The existing models do not explain this complete picture portrayed here and the processing steps have also not been stated or considered.

This proposed architecture aspires to give this top level view of information integration with SOA that could be adopted are made suitable to any information integration problem. The proposed architecture is an abstract one that can be realized as a frame work and made concrete when applied on a domain or application or problem. The proposed architecture for information integration using SOA is shown below figure.

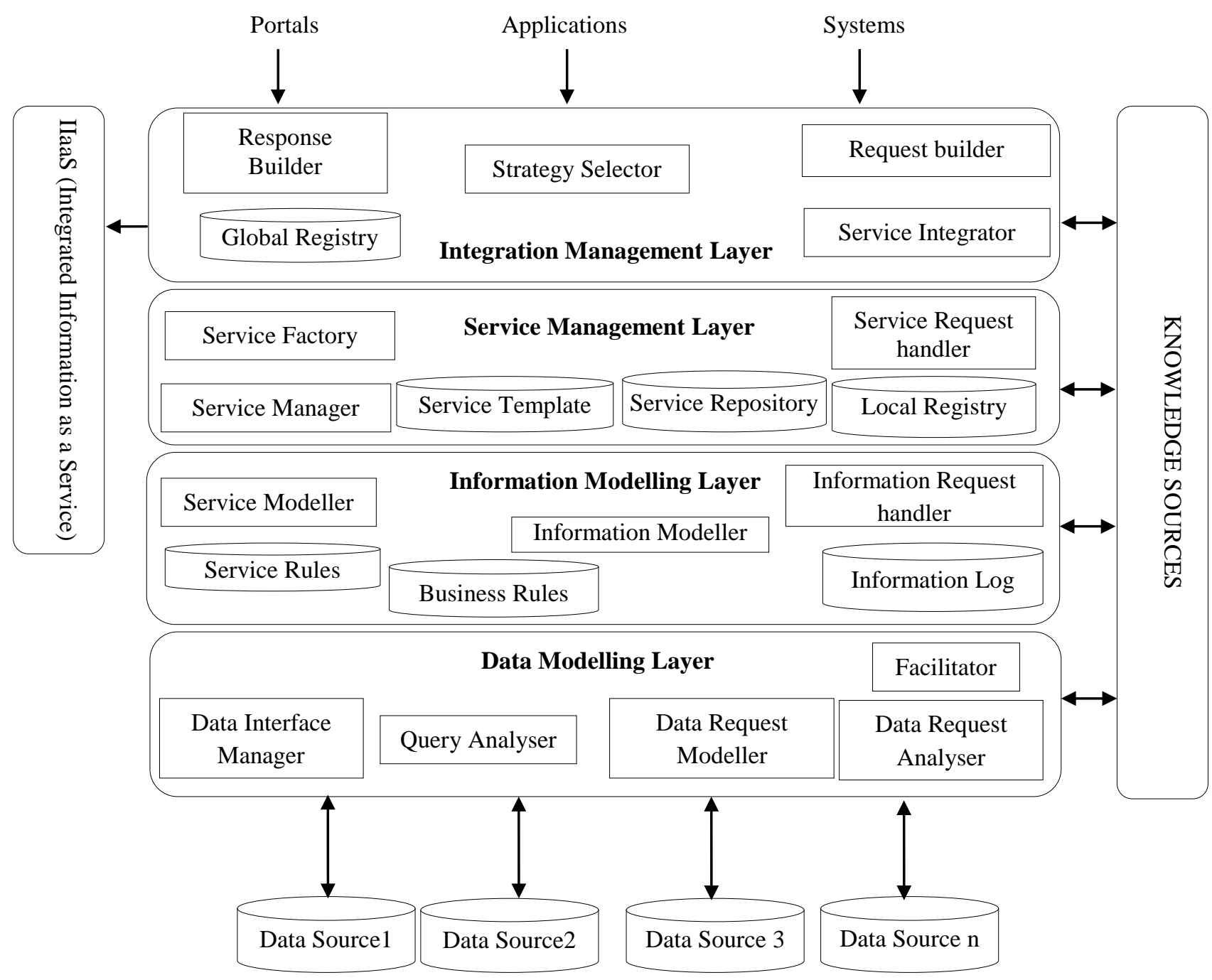

Fig 1. Information Integration Architecture

Four layers namely the data modelling layer, information modelling layer, service management layer, and integration management layer are designed to organize the components of information integration. These layers are segregated as per the major functionalities in accordance to the activities specified for information integration and the components are correspondingly grouped. This stands out as an enhanced where the layers are along with all of its functionalities are realized as components to form architecture. The information request takes many forms as it gets processed, as service request, information request and data request respectively in each of the layer to be passed to the next layer. The request travels in a top down fashion. The request when converted into response travels upward on being formulated into different forms like modelled data, candidate service, and information service and then integrated information as service from layer to 
layer. The roles and responsibilities of each layer are exposed here to give a precise understanding and significance of the layers. The components are also explained with respect to a template and thereby the complete architecture is put forth in this section.

Components are designed based on the functional requirements of information integration architecture using SOA. Each component reflects one or more functionalities and is grouped onto respective layers. Components are explained with respect to a template. This template gives the basic structure and purpose of the components. The attributes of this template are aimed at providing the essential aspects of the component in this architecture. It states the need of the component and signifies the presence of it with respect to the architecture.

\section{Component Name \\ Intent \\ Functions \\ Input \\ Output \\ Collaborations \\ Dependencies}

Component Name - The name of the component Intent: Refers to the main purpose of the component Functions: States the processes it does in order to achieve the purpose of the component

Input: Indicates what is and type of input the component receives

Output: Gives the result of all processing that happens in the component

Collaborations: Specify the other components to which this component communicates

Dependencies: Refers to other data sources, knowledge bases or registries that support in implementing and obtaining the result

\subsection{The Layers and Components Description}

\section{Integration Management Layer:}

This is topmost layer which provides the interface between this architecture and the initiator be it application or system or device or stakeholder who is in need of some information that lies across sources in different forms. Service oriented Integration is major concentration of this layer and the roles and responsibilities towards this are specified, along with description of the associated components.

Roles: Application/portal/system request handling, service integration and response building with respect to the request.

\section{Responsibilities:}

- Any request is analyzed with the domain knowledge for it to be broken down into one or more information associated with it.

- The information is requested as information service request to the lower layer.

- The services are to be integrated depending upon their type and agreements.

- The resultant of service integration is also to be provided as service.
- The response or the result is formulated and presented as requested by the requestor.

- Maintain the service registry.

Components:

Component Name: Request builder

Intent: To structure and interpret the request to bring out the necessary information services to be built

Functions:

- To understand the context of the request

- To decompose the request into the respective elements with respect to domain knowledge

- To organize the request

- To pass the request to the Service request handler pertaining to the lower layer

Input: Request

Output: Structured Request

Collaborations: Strategy selector, Service request handler

Dependencies: Domain Ontology, Global Registry

Component Name: Strategy Selector

Intent: To provide the respective plan for information services integration

Functions:

- Maintains the various information that is processed from the request

- Checks for the availability of the needed information service in the service registry.

- Formulates the workflow of finding all the information associated with the request and later on to be integrated to provide the result or response.

- Pass the information that is available as services to the integrator.

Input: The interpreted request from the system request handler Output: Integrated information as a service.

Collaborations: Request Handler, Service Integrator

Dependencies: Domain Ontology, Service Registry.

Component Name: Service Integrator

Intent: To integrate services pertaining to the strategy

adopted.

Functions:

- To provide interaction and interoperability between services

- Check for adherence to service principles and the strategy adopted while integration.

Input: The information services that need to be integrated

Output: The integrated service

Collaborations: Strategy selector, Response builder

Dependencies: nil

Component Name: Response Builder

Intent: To provide the reply to the system request Functions:

- To formulate the response with respect to the request

- To structure the reply as the need of the initiator

Input: The integrated service

Output: Intended information

Collaborations: Request handler, Service Integrator

Dependencies: Domain knowledge

\section{Service Management Layer}

This layer handles the service creation and management. It receives the structured request, processes it to be derived into services request and passes to the information layer. The response receives from the information layer is a candidate service, which is processed in this layer. Stated below are the 
roles, responsibilities and thereby the respective components of this layer.

Roles: Information service request handling, designing of service contract, service creation

Responsibilities:

- The information request is processed for the information to be broken up into various service requests.

- The candidate service is assigned with the associated service template

- Services are stated with service rules stating the ownership and usage rights

- Maintains the service with respect to usage and changes

- Creates a service and registers it into the registry.

\section{Components}

Component Name: Service Request Handler

Intent: To structure and interpret the request to find out the necessary service request associated with the initial request.

Functions:

- To understand the context of the information service request

- To decompose the request into the respective information service elements

- To organize the request as per information services needed

- To pass the request to the information request handler pertaining to the lower layer

Input: Structured Request

Output: Information Service Request

Collaborations: Service manager,

Dependencies: Domain Ontology, local registry

Component Name: Service Manager

Intent: To assign ownership rights in order to maintain the service

Functions:

- To state the owner of the service

- Incorporates the access rights to the service

- Provide the scope of the service

Input: Newly created Service

Output: Owned Service

Collaborations: Service Factory

Dependencies: Service repository, service rules

Component Name: Service Factory

Intent: To create a service

Functions:

- To create a service interface, and link to implementation

- To change candidate operations into service functionalities

- Create a service with respect to the language template

- Created service to be published in service registry Input: Candidate Service along with candidate operations. Output: New Service

Collaborations: Service Manager, Service modeller Dependencies: Service repository, Local registry, Service Template

\section{Information Modelling Layer}

Modelled data are made to be converted into information along with the access rights, security aspects and also separation of interface from implementation are the core functionalities of this layer. This layer initiates the process of information to be represented as service. The details of the various functionalities are expressed as roles, responsibilities and components below.

Roles: service request handling for respective information request, information modelling and service modelling.

Responsibilities:

- The request is an information request i.e., the information that needs to be converted into service and integrated

- $\quad$ The request is processed with domain knowledge and business rules for it to be broken into data request.

- The data received as response is modelled and converted into information and thereby for it to be converted into a service

- Add policies and update write policies for reuse.

- Information procedures are modelled as service operations

- $\quad$ Service policies and contracts, service level agreements are stated.

- Candidate service and candidate service operations are passed to the service layer.

Components

Component Name: Information Request Handler

Intent: To structure and interpret the request to find out the necessary information request associated with the information service request.

Functions:

- To understand the context of the information request

- To decompose the request into the respective information elements

- To organize the request as per information needed

- To pass the request to the data request analyzer pertaining to the lower layer

Input: Service Request

Output: Structured Information Reques

Collaborations: Service manager,

Dependencies: Domain Ontology, Information log, Business rules

Component Name: Service Modeller

Intent: To build candidate service and candidate service operations

Functions:

- Break down the documented information into granular process

- Decompose the process workflow of information obtained into series of process steps

Input: Information

Output: Candidate service, Service candidate operations

Collaborations: Information modeller, Service factory

Dependencies: Service rules

Component Name: Information Modeller Intent: To organize the data into information

Functions:

- Transform data from one format to another.

- To access data it has to be organized or re-organized according to database management rules and procedures.

- Changing the format of the data to acceptable form for software application or information system

Input: Data retrieved from multiple data sources

Output: Organized/ structured Information

Collaborations: Facilitator, Service modeller 
Dependencies: Domain Knowledge, Business rules, information log.

\section{Data Modelling Layer:}

The information request when it reaches this layer comes as data request, that is, the associated data elements that are needed to form the information. This layer facilitates between the request, query formulation and data retrieval from the data sources. This is where the underlying data for the requested information is obtained and restructured. The roles, responsibilities, and components that assist in bring out the required aspects of this layer are given below.

Roles: Data request to be analyzed with the domain ontology, business policies for the data retrieval, query processing, data source interaction, data federation

Responsibilities:

- To interpret the request for necessary data with respect to ontology

- To structure the request with respect to business context and system format

- To form specific and sub queries to retrieve the data from the respective data sources

- To facilitate between heterogeneous data sources and acts as a interface manager for the data sources.

\section{Components}

Component Name: Data Request Analyzer

Intent: To structure and interpret the information request to find out the necessary data associated with the information request.

Functions:

- To understand the context of the data request

- To decompose the request into the respective data elements

- To organize the request as per data needed

- To pass the request to the data request modeller to form queries

Input: Data Request

Output: Data

Collaborations: Facilitator, Data Modeller

Dependencies: Domain Ontology

\section{Component Name: Facilitator}

Intent: To coordinate the data request for it to be converted into corresponding data

Functions:

- To send the data request and get it modelled from the request modeller

- To communicate with the query analyser for the processed query corresponding to the data.

- To organize the query with respect to the data source

- To communicate with the data base interface manager and retrieve the data

- To restructures and combines the data from various sources into one data

- It's a co-routine where simultaneously it could be executed for several requests into various servers.

Input: Data request

Output: Requested data

Collaborations: Request modeller, Query analyzer, Database interface manager, Data request analyzer

Dependencies: nil

Component Name: Request Modeller Intent: To structure the request
- To convert the data request into a specified format

- To check for access rights and various implementations constrains

Input: Data request

Output: Structured request

Collaborations: Facilitator

Dependencies: Domain data sources ontology, access rules

Component Name: Query Analyzer

Intent: To form the abstract queries and sub queries

Functions:

- To interpret the request and generate an execution plan for processing the request

- The query compiler uses a language-specific lexical analyzer and parser to interpret the request and populate a structure

- The Query analyzer passes the plan to facilitator for the execution of the query

Input: Structured Data request

Output: Abstract queries and sub queries

Collaborations: Facilitator

Dependencies: Nil

Component Name: Database Interface Manager

Intent: To access the needed data sources and retrieve

the data

Functions:

- Change the abstract queries into concrete query with respect to the data sources

- Access the data source and retrieve the data

- Pass the data to the facilitator

Collaborations: Facilitator

Dependencies: Data sources 1, Data sources 2,..., Data source

The knowledge sources pertain to the semantics, ontology and business context of the domain. These form the basic backbone of the architecture, where all the components function based on this. The information sources are also assumed to be of all types' structured, semi structured and unstructured. The users of this architecture are any application, or portals or device or mangers etc.

\section{DISCUSSION}

The proposed architecture has given a complete picture of information integration covering all the aspects of Input, Output and Process. To bring out its efficiency it has to be analyzed with other such models. In general if an architecture is built it is scrutinized to posses certain characteristics to satisfy its intent. Such characteristics that need to be addressed by architecture are listed out as row heading in the table below and each column specify a research work. The existing research work stated in the related work comprises of different works of information integration.

Generalness: This attributes indicates whether the model or architecture of framework is general or specific. General indicates that the components are built to portray the common functionalities with the associated constraints; specific architecture is one which adopts certain constraints of a particular purpose with respect to a specific domain.

Completeness: This refers to the whether the architecture has addressed all the functionalities of information integration as described in the proposed architecture.

Openness: It indicates the nature of the architecture and components that relates to being proprietary or open. Functions: 
Data Source: This attribute refers to the data sources the architecture has considered; Structured, semi structured and Unstructured.

Integration: The integration states whether the architecture has addressed data integration or information integration.
Software Qualities: The non-functional qualities addressed by the architecture have been indicated through this attribute.

Output: The resultant output of the architecture is information or service.

Implementation Types: the architecture or its components adheres to specific standards or left open is shown here

Table 1. Comparison of Information Integration Architectures

\begin{tabular}{|c|c|c|c|c|c|c|c|c|}
\hline Architectures & Generalness & $\begin{array}{c}\text { Components } \\
\text { Type }\end{array}$ & $\begin{array}{c}\text { Data } \\
\text { Source }\end{array}$ & Integration & Outcome & Qualities & $\begin{array}{c}\text { Implementation } \\
\text { types }\end{array}$ & Completeness \\
\hline Aridane & $\begin{array}{c}\text { Web Sources } \\
\text { Centric }\end{array}$ & Open & $\begin{array}{c}\text { Web } \\
\text { Sources }\end{array}$ & Information & $\begin{array}{c}\text { Informatio } \\
\mathrm{n} \text { or web } \\
\text { reference }\end{array}$ & no & $\begin{array}{c}\text { Incremental } \\
\text { development, } \\
\text { machine learning, } \\
\text { mediator based }\end{array}$ & $\begin{array}{c}\text { Mapping of web } \\
\text { sources }\end{array}$ \\
\hline MetaQuerier & $\begin{array}{l}\text { Web sources } \\
\text { Centric }\end{array}$ & Open & \begin{tabular}{|c|} 
Deep \\
Web or \\
Web \\
Sources \\
\end{tabular} & $\begin{array}{l}\text { Information } \\
\text { with } \\
\text { semantics }\end{array}$ & $\begin{array}{c}\text { Informatio } \\
\mathrm{n}\end{array}$ & \begin{tabular}{|l|} 
Dynamic, \\
On the fly \\
integration
\end{tabular} & $\begin{array}{l}\text { Cascading, accepts } \\
\text { action feedback }\end{array}$ & Integration only \\
\hline InfoMosaic & $\begin{array}{c}\text { Query and } \\
\text { Source Centric }\end{array}$ & & $\begin{array}{c}\text { Multi } \\
\text { domain } \\
\& \text { Geo } \\
\text { spatial } \\
\text { data }\end{array}$ & $\begin{array}{c}\text { Data } \\
\text { Integration }\end{array}$ & $\begin{array}{c}\text { Informatio } \\
\mathrm{n}\end{array}$ & $\begin{array}{l}\text { Result } \\
\text { Ranking }\end{array}$ & $\begin{array}{c}\text { XQueries \& XML } \\
\text { Repository }\end{array}$ & $\begin{array}{c}\text { Data Modelling } \\
\text { layer. }\end{array}$ \\
\hline $\begin{array}{l}\text { Automated } \\
\text { ontology }\end{array}$ & $\begin{array}{c}\text { Ontology and } \\
\text { Semantic } \\
\text { centric } \\
\end{array}$ & Proprietary & $\begin{array}{c}\text { Data as a } \\
\text { service }\end{array}$ & $\begin{array}{c}\text { Data Service } \\
\text { Integration }\end{array}$ & $\begin{array}{l}\text { Web } \\
\text { Service }\end{array}$ & Automation & $\begin{array}{c}\text { SparQL } \\
\text { datasources, OWL }\end{array}$ & $\begin{array}{l}\text { Integration } \\
\text { engine }\end{array}$ \\
\hline Searchy & $\begin{array}{c}\text { Ontology and } \\
\text { Web service } \\
\text { Centric }\end{array}$ & Open & $\begin{array}{c}\text { Digital } \\
\text { Informati } \\
\text { on } \\
\text { Sources }\end{array}$ & Information & Message & \begin{tabular}{|c|} 
Information \\
source \\
Scalability, \\
Loose \\
coupling \\
\end{tabular} & $\begin{array}{c}\text { OWL/RDFS, MTS, } \\
\text { FIPA }\end{array}$ & Integration \\
\hline $\begin{array}{l}\text { Workflow } \\
\text { Based } \\
\text { Informatin } \\
\text { Integration } \\
\text { (WII) }\end{array}$ & $\begin{array}{l}\text { Work plan } \\
\text { centric }\end{array}$ & Proprietary & $\begin{array}{l}\text { Sources } \\
\text { as web } \\
\text { service }\end{array}$ & $\begin{array}{c}\text { Data } \\
\text { Services }\end{array}$ & Work flow & Global view & Web Service & All layers \\
\hline Loglo & $\begin{array}{c}\text { Provider } \\
\text { registry and } \\
\text { Consumer } \\
\text { Based } \\
\end{array}$ & Proprietary & $\begin{array}{c}\text { Heteroge } \\
\text { nous } \\
\text { source }\end{array}$ & Information & $\begin{array}{c}\text { Informatio } \\
\mathrm{n}\end{array}$ & \begin{tabular}{|c|} 
Large Scale \\
Integration, \\
Loose \\
coupling \\
\end{tabular} & REML, XML, & Prototype \\
\hline $\begin{array}{c}\text { Online } \\
\text { Accommodatio } \\
n\end{array}$ & $\begin{array}{l}\text { Ontology - } \\
\text { based \& } \\
\text { Semantic }\end{array}$ & Open & $\begin{array}{l}\text { Web } \\
\text { portals }\end{array}$ & Information & $\begin{array}{c}\text { Web based } \\
\text { informatio } \\
n\end{array}$ & \begin{tabular}{|c|} 
Hybrid \\
Integration, \\
Dynamic \\
inclusion of \\
sources \\
\end{tabular} & $\begin{array}{l}\text { XML, Schema } \\
\text { Mapping }\end{array}$ & Prototype \\
\hline $\begin{array}{c}\text { Personalisation } \\
\text { for Higher } \\
\text { Education }\end{array}$ & $\begin{array}{c}\text { Semantic and } \\
\text { Web Service } \\
\text { Centric }\end{array}$ & Open & \begin{tabular}{|c|} 
Higher \\
Educatio \\
$\mathrm{n}$ \\
Informati \\
on \\
Sources \\
\end{tabular} & Information & Service & $\begin{array}{l}\text { On-demand } \\
\text { integration }\end{array}$ & $\begin{array}{l}\text { Web Services } \\
\text { Standards }\end{array}$ & All Layers \\
\hline $\begin{array}{c}\text { Information } \\
\text { Fabric } 3.0\end{array}$ & \begin{tabular}{|c|} 
Data \\
Virtualisation \\
Centric
\end{tabular} & Open & $\begin{array}{c}\text { Any } \\
\text { distribute } \\
\mathrm{d}, \\
\text { hetrogen } \\
\text { eous } \\
\text { Sources } \\
\end{array}$ & Data & View & \begin{tabular}{|} 
Reference \\
Model, Real \\
time \\
Information
\end{tabular} & Not Specified & $\begin{array}{l}\text { Data Modelling } \\
\text { and Service } \\
\text { Management } \\
\text { Layer. }\end{array}$ \\
\hline $\begin{array}{l}\text { Proposed } \\
\text { Architecture }\end{array}$ & Generic & Open & $\begin{array}{c}\text { Any type } \\
\text { Sources }\end{array}$ & Information & \begin{tabular}{|l|} 
Integrated \\
Informatio \\
$n$ as service \\
\end{tabular} & $\begin{array}{l}\text { Dynamic, } \\
\text { Automated, }\end{array}$ & Open & All Layers \\
\hline
\end{tabular}

The above table gives details about the various characteristics of the models or architectures indicated in the related work. This implies to the details specified in the above mentioned limitation. Each model in one way or other doesn't consider all aspects of information integration, instead designed to solve one or more functionalities intended for.

Hence, the proposed architecture satisfies all the characteristics. It does not address specific issues but the 
whole information integration process, the components are not tied to the architecture and depending on the need any layer could be considered. The sources are not limited; all type of data sources distributed geographically is considered and handled appropriately. The result information is provided as a service where it is structured as per the request along with its access rights and security mechanisms. This Proposed architecture addresses all layers information integration in complete starting from handling the request, to data retrieval, conversion \& modelling, service creation and Management, integration of information service and to building the response and presenting to the requestor of this architecture. These layers and components could be standardised and used for any information integration problem.

\section{CONCLUSION}

Information integration has been brought out through various approaches, architectures, frameworks etc., but these solutions do not give a complete picture. Access to enormous information lies without any suitable theory, technology, or tools to be used in an appropriate fashion to obtain clear and needed information. Information integration should result in producing right information in the right form so that it could enable them to perform efficient decision making or any relevant purpose. This has been the motive of this research and architecture has been designed to give a clear and big picture comprising all aspects of information integration seamlessly. The architecture and the components are explained, where the execution flow and evaluation of the architecture is still in the research progress.

\section{REFERENCES}

[1] Alon Y. Halevy, Naveen Ashishy, Dina Bittonz, Michael Careyx, Denise Draper, Jeff Pollockk, Arnon Rosenthal, Vishal Sikkayy, "Enterprise Information Integration: Successes, Challenges and Controversies", ACM SIGMOD, June 2005 .

[2] "What Is Enterprise Information Integration (EII)?", Gilbane Report, volume 12, No. 6, 2004.

[3] Kai Yang, Robert Steele, A Semantic Integration Solution for Online Accommodation Information Integration, 978-1-4244-8756-1, IEEE, 2011

[4] Bahram Amini, Roliana Ibrahim, Mohd Shahizan Othman, A Framework for Personalized Information Integration in Higher Education Institutes, International Journal of Computer Applications (0975 - 8887), Volume 23- No.4, June 2011

[5] Diego Calvanese, Giuseppe De Giacomo, Maurizio Lenzerini, Daniele Nardi, Riccardo Rosati, Information Integration:Conceptual Modeling and Reasoning Support, 3rd IFCIS, International Conference on Cooperative Information Systems, Pgs 280-289, Aug 1998

[6] Jaime A Reinoso Castillo, Adrian Silvescu, Doina Caragea, Jyotishman Pathak, Vasant G Honavar, Information Extraction and Integration from Heterogeneous, Distributed, Autonomous Information Sources - A Federated Ontology-Driven Query-Centric Approach, IEEE International Conference on Information Reuse and Integration, 2003.
[7] Oliver M. Duschka, Michael R. Genesereth, InfomasterAn Information Integration Tool, International Workshop on Intelligent Integration, Friedurg, Germany, 1997.

[8] Bostjan Grasic, Vili Podgorelec, Automating Ontology Based Information Integration Using Service Orientation,Wseas Transactions On Computers,Issn: 1109-2750 547 Issue 6, Volume 9, June 2010.

[9] David f. Barrero, mar' ia d. R-moreno, Information Integration In Searchy: An Ontology and Web Services Based Approach, International Journal of Computer Science and Applications,Vol. 7 No. 2, pp. 14 - 29, 2010.

[10] Semantic Web Services in Action - Enterprise Information Integration,B. Krämer, K.-J. Lin, and P. Narasimhan (Eds.): ICSOC 2007, Springer-Verlag LNCS 4749, pp. 485-496

[11] Song Xiao, Zhang Lin, Yu Peng-fei,Semantic SOABased Enterprise Information System Integration Technology, International Conference on Smart Manufacturing Application, April. 9-11, 2008 in KINTEX, Gyeonggi-do, Korea.

[12] Craig a. Knoblock, Steven minton, Jose luis ambite, Naveen ashish, Ion muslea, Andrew g. Philpot, and Sheila tejada,The aridane approach to web-based information integration, International Journal of Cooperative Information Systems, World Scientific Publishing Company, 2001.

[13] Toward Large Scale Integration: Building a MetaQuerier over Databases on the Web. K. C.-C. Chang, B. He, and Z. Zhang. In Proceedings of the Second Conference on Innovative Data Systems Research (CIDR 2005), Asilomar, California, January 2005.

[14] Aditya Telang, Sharma Chakravarthy, Information Integration across heterogeneous domains: current scenario, challenges, and the InfoMosaic Approach, Technical Report CSE-2007, University of Texas.

[15] X.Y. Li, Y. Qian, AWEbservice Based Enterprise Information Integration Model, Proceedings of 4th International Conference on Computer Science and Education, 978-1-4244-3251-0, IEEE, 2009.

[16] Xiangyu Li, An Integration Framework for Information System based on Web Service, 978-1-4244-5265-1, IEEE, 2010

[17] Patrick C.K. Hung, Dickson K.V.Chiu, Devolping Workflow-based Information Integration with Exception Support in a Web Services Environment, Proccedings of the $37^{\text {th }}$ Hawaii International Conference on System Sciences,IEEE, 2004.

[18] Feng Baiming, Ma Manfu, Gou Heping, An Architecture to Integrate the Distributed Heterogenous Information, Eighth International Conference on Grid and Cooperative Compouting, IEEE, 2009.

[19] Shuwang Ke, Jitao Fang, XiangqianDing,Shusong Yu, Dan Li, The Design and Development of Shop-level Information Integration System, International Conference on Electrical and Control Engineering, 978-0-7695-40313, IEEE, 2010.

[20] Yong-Gang Gong, Xin Chen, Healthcare Information Integration and Shared Platform Based on ServiceOriented Architectures,2nd International Conference on 
Signal Processing Systems (ICSPS), 978-1-4244-6893, IEEE, 2010.

[21] Haiyan Chen, Shigang Qin, Jianxun Liu, Jian Cao, SOAEnabled Health Information Integration Platform (HIIP): A Case Study, Fifth International Conference on Semantics, Knowledge, Grid, 978-0-7695-3810-5, IEEE, 2009.

[22] Brian Hopkins, Alex Cullen, Mike Gilpin, Boris Evelson, Gene Leganza, and Mackenzie Cahill, Data Virtualization Reaches Critical Mass, Forrester Report, 2011.

[23] Noel Yuhanna and Mike Gilpin, Information Fabric 3.0, Forrester Report, 2013.
[24] Laura Haas, Beauty and the Beast: The Theory and Practice of Information Integration ICDT 2007 , Vol. 4353 (2006), pp. 28-43.

[25] Laura M. Haas, Aya Soffer, New Challenges in Information Integration, DaWaK 2009, Springer-verlag LNCS 5691, pp.1-8, 2009.

[26] C.Punitha Devi, V. Prasanna Venkatesan , G. Shanmugasundaram, Research Opportunities in Information Integration, AICTE sponsored National conference on Internet and Web Service Computing (NCIWSC 2012), Pondicherry Engineering College, 2012.

[27] C.Punitha Devi, V. Prasanna Venkatesan , G. Shanmugasundaram, Information Integration Redefined, ARPN Journal of Systems and Software, Vol 2, No:4, 2012 , ISSN 2222-9833 